\title{
Estimativas das componentes da radiação solar incidente em superfícies inclinadas baseadas na radiação global horizontal
}

\author{
Adilson P. Souza ${ }^{1}$, João F. Escobedo ${ }^{2}$, Alexandre D al Pai ${ }^{3}$ \& Eduardo N. G omes ${ }^{2}$
}

\section{RESU MO}

Foram avaliadas equações estatísticas de estimativas com agrupamentos de dados anuais e mensais e suas respectivas validações, para as componentes global, direta e difusa da radiação solar incidente em superfícies inclinadas a $12,85,22,85$ e 32,85ㅇ, com face para o $\mathrm{N}$ orte, nas condições climáticas e geográficas de Botucatu, SP. Empregou-se as frações das três componentes da radiação a do topo da atmosfera em correlação com o coeficiente de transmissividade atmosférica do plano horizontal, em uma base de dados de abril/1998 a dezembro/2007, cujas medidas nas três inclinações ocorreram em diferentes períodos, todavia concomitantes ao plano horizontal. 0 aumento do ângulo de inclinação da superfície propiciou aumento do espalhamento dos valores diários do índice de claridade para superfícies inclinada e horizontal. N os agrupamentos anuais os piores desempenhos foram verificados na estimativa da radiação difusa diária para superfície inclinada, com valores máximos de espalhamentos iguais a $3,89 \mathrm{MJ} \mathrm{m}^{-2} \mathrm{~d}^{-1}(43,65 \%)$ e ajustamento em torno de $62 \%$. Na estimativa das componentes global e direta da radiação solar nos planos inclinados, podem ser aplicadas, tanto as equações anuais como as mensais, com desempenhos dependentes das condições climáticas.

Palavras-chave: índice de claridade, indicativos estatísticos, modelagem

\section{Estimates of solar radiation components on a tilted surface based on global horizontal radiation}

\begin{abstract}
Statistics equations and validations with groups of annual and monthly data were evaluated for global, direct and diffuse solar radiation components incident on the tilted surface to $12.85,22.85$ and $32.85^{\circ}$ with the face $\mathrm{N}$ orth, in climate and geographical conditions of Botucatu, SP. It was employed the fractions of three components of extraterrestrial radiation in correlation with the coefficient clearness index horizontal plane, in a database of April/1998 to December/2007, whose measures at different periods in three inclinations, how ever concomitant to the horizontal plane. Increasing the angle of the surface led to increased scattering of the daily values of clearness index for inclined and horizontal surfaces. In annual groups, the lower performances were observed in the estimation of inclined daily diffuse radiation, with maximum Root Mean Square Error to $3.89 \mathrm{MJ} \mathrm{m}^{-2} \mathrm{~d}^{-1}(43.65 \%)$ and adjustments around $62 \%$. In estimates of global and direct components of solar radiation on inclined planes, both annual and monthly equations can be applied, with performance dependents to climatic conditions.
\end{abstract}

Key words: clearness index, indicate estatistical, modeling 


\section{INTRODUÇÃO}

A crescente demanda por informações sobre a utilização da radiação solar como fonte suplementar e alternativa de energia predispõe a necessidade de conhecimentos diferenciados sobre a incidência da radiação solar e sobre superfícies com diferentes topografias naturais ou induzidas.

Qualquer aplicação de uso da energia solar deve começar pelo estudo das variações ao longo do ano, em termos regionais e para diferentes condições de exposição. Para Scolar et al. (2003), estudos das intensidades e variações de radiação solar devem compreender medidas por um período mínimo de um ano. As séries temporais e espaciais das componentes da radiação incidente à superfície, permitem conhecer a disponibilidade energética diurna, mensal e anual; entretanto, exigem medidas simultâneas de rotina em diferentes condições astronômicas, geográficas e climáticas.

A maioria das estações meteorológicas mede, de forma rotineira, apenas a radiação solar global em planos horizontais, enquanto medidas das componentes direta e difusa são escassas em razão dos elevados custos dos métodos e instrumentos envolvidos. Entraves, como a dificuldade de manutenção e obtenção de uma rede solarimétrica que permita gerar informações sobre radiação solar em toda a extensão continental, ainda são evidentes. O monitoramento e medições rotineiras são restritas às Universidades (Pereira et al., 1998; Souza et al., 2005; Tiba et al., 2005; Gomes et al., 2006; Codato et al., 2008; Borges et al., 2010; Silva et al., 2010) e realizadas com apenas uma ou duas das partições da radiação solar inviabilizando, assim, o estudo variacional, até mesmo para uma única região.

$\mathrm{Na}$ estimativa da radiação solar normalmente são empregados dois tipos de modelo: estatísticos (ou de decomposição) e paramétricos. Os modelos paramétricos carecem de informações mais detalhadas das condições atmosféricas locais, conforme a componente a ser estimada, ou então a utilização de percentuais fixos de ocorrência de cada componente da radiação solar global (Liu \& Jordan, 1960; Turco \& Rizzatti, 2006; Notton et al., 2006; Jiang, 2009). Os modelos estatísticos são representados por equações matemáticas ajustadas por meio de regressão linear ou polinomial em correlações com as frações radiométricas ou com a razão de insolação, caso em que se torna, necessária a verificação do desempenho desses modelos com base em comparações de valores estimados com dados medidos não empregados na obtenção das equações.

O procedimento mais comum citado na literatura, é a estimativa das componentes direta e difusa, a partir de valores medidos ou estimados da radiação global (Liu \& Jordan, 1960). Como as medidas em superfícies inclinadas também não são rotineiras no Brasil, é pertinente que essas correlações sejam feitas com base na radiação global medida no plano horizontal permitindo, assim, estimar as componentes global, direta e difusa da radiação solar em uma superfície inclinada com base em medidas na horizontal.

Considerando a necessidade do conhecimento da radiação solar incidente em superfícies inclinadas para muitas áreas de pesquisa em atividade e desenvolvimento no Brasil, o presente trabalho objetivou propor correlações entre as frações das componentes da radiação solar global, direta e difusa em superfícies inclinadas a $12,85,22,85$ e $32,85^{\circ}$ e o índice de claridade no plano horizontal, nas escalas anual e mensal, para a região de Botucatu, SP.

\section{MATERIAL E MÉTODOS}

Os dados instantâneos das componentes global, direta e difusa da radiação solar, foram coletados na Estação de Radiometria Solar da UNESP de Botucatu, SP (latitude 22 $2^{\circ}$ ', S; longitude $48^{\circ} 26^{\prime} \mathrm{W}$; altitude $786 \mathrm{~m}$ ), no período de abril de 1998 a dezembro de 2007. De acordo com a classificação climática de Köppen, o clima da região de Botucatu é classificado como Cwa, caracterizado como temperado úmido, com inverno seco e verão quente (CEPAGRI, 2009).

As medidas das componentes supracitadas nos três planos inclinados a $12,85^{\circ}$ (latitude local $+10^{\circ}$ ), $22,85^{\circ}$ (latitude local) e $32,85^{\circ}$ (latitude local $-10^{\circ}$ ) ocorreram em diferentes períodos: entre 09/2001 e 02/2003, 04/1998 e 08/2001, 03/2004 e 12/2007, respectivamente. Esses dados sofreram uma análise de consistência e os valores discrepantes (oriundos de erros de leitura dos sensores ou mau funcionamento do sistema de aquisição de dados) foram removidos das séries. Para a geração dos modelos de estimativa estatística usaram-se dados de 2 anos para as inclinações de 22,85 e $32,85^{\circ}$ e de um ano para $12,85^{\circ}$, sendo os demais dados em cada inclinação empregados nas validações desses modelos. Tais modelos foram gerados com agrupamentos de dados mensais e anuais, com base na correlação entre as frações das três componentes da radiação estudadas.

As leituras foram feitas a cada segundo e as médias armazenadas em intervalos de 5 minutos, as quais foram consideradas valores instantâneos. Utilizou-se um sistema de aquisição de dados Microlloger Campbell Scientific, Inc., modelo CR23X, operando na frequência de $1 \mathrm{~Hz}$; na transferência dos dados foi empregado um módulo de memória SM192, também da Campbell, com interface SC532 para microcomputador operado pelo software PC 208W.

A radiação solar global instantânea no plano horizontal $\left\langle\mathrm{I}_{\mathrm{GH}}\right\rangle$ foi medida com piranômetro Eppley-PSP, com fator de calibração de 7,45 $\mu \mathrm{V} \mathrm{W}^{-1} \mathrm{~m}^{-2}$ e linearidade de $\pm 0,5 \%$ ( 0 a 2800 $\mathrm{W} \mathrm{m} \mathrm{m}^{-2}$. Para a radiação global instantânea nas superfícies inclinadas $\left\langle\mathrm{I}_{\mathrm{G} \beta}\right\rangle$, os piranômetros foram posicionados paralelamente sobre planos inclinados a $12,45^{\circ}, 22,45^{\circ}$ e $32,45^{\circ}$ com a face voltada para o Norte. A radiação solar direta instantânea na incidência $\left\langle\mathrm{I}_{\mathrm{BN}}\right\rangle$ foi obtida por um pireliômetro Eppley-NIP, acoplado a um rastreador solar ST3 EPPLEY, com fator de calibração de $7,59 \mu \mathrm{V} \mathrm{W}^{-1} \mathrm{~m}^{-2}$ e linearidade de $\pm 0,5 \%$ ( 0 a $1400 \mathrm{~W} \mathrm{~m}^{-2}$ ).

Os valores da radiação solar global diária horizontal $\left\langle\mathrm{H}_{\mathrm{GH}}^{\mathrm{d}}\right\rangle$, inclinada $\left\langle\mathrm{H}_{\mathrm{G} \beta}^{\mathrm{d}}\right\rangle$ e direta na incidência normal $\left\langle\mathrm{H}_{\mathrm{BN}}^{\mathrm{d}}\right\rangle$, foram obtidos através da integração das respectivas curvas de radiação instantânea, integrada do nascer ao pôr do sol. A projeção da radiação direta diária na horizontal $\left\langle\mathrm{H}_{\mathrm{BH}}^{\mathrm{d}}\right\rangle$ foi dada pelo produto entre $\left\langle\mathrm{H}_{\mathrm{BN}}^{\mathrm{d}}\right\rangle$ e o cosseno do ângulo zenital diário (Iqbal, 1983). Nas superfícies inclinadas a radiação direta diária foi obtida pela aplicação do fator geométrico $\left\langle\mathrm{R}_{\mathrm{B}}\right\rangle$, que traduz a relação entre a radiação no topo da atmosfera para a superfície 
inclinada e para a superfície horizontal (Simonson, 1981; Iqbal, 1983; Wenxian et al., 1995).

A radiação difusa diária incidente no plano horizontal foi obtida pela diferença entre $\left\langle\mathrm{H}_{\mathrm{GH}}^{\mathrm{d}}\right\rangle$ e $\left\langle\mathrm{H}_{\mathrm{BH}}^{\mathrm{d}}\right\rangle$; já no plano inclinado, a radiação difusa também foi obtida por diferença entre as componentes global, direta e refletida da radiação solar. Esta componente refletida diária da radiação global incidente em superfícies inclinadas foi considerada apenas com comportamento isotrópico (Iqbal, 1983; Wenxian et al., 1995; Kamali et al., 2006; Gueymard, 2009). A radiação solar diária incidente no topo da atmosfera para as superfícies horizontal $\left\langle\mathrm{H}_{0 \mathrm{H}}^{\mathrm{d}}\right\rangle$ e inclinada $\left\langle\mathrm{H}_{0 \beta}^{\mathrm{d}}\right\rangle$ foi estimada segundo Iqbal (1983).

Os índices diários de claridade $\left\langle\mathrm{K}_{\mathrm{T}}^{\mathrm{d}}\right\rangle$ foram obtidos pela razão entre a radiação global $\left\langle\mathrm{H}_{\mathrm{G}}^{\mathrm{d}}\right\rangle$ e a radiação no topo da atmosfera $\left\langle\mathrm{H}_{0}^{\mathrm{d}}\right\rangle$, para superfícies inclinadas e horizontais, para os mesmos períodos de medidas.

Como na grande maioria das estações da rede meteorológica brasileira, até mesmo medidas da radiação global em planos inclinados são escassas, nas correlações para geração dos modelos de estimativa das radiações direta e difusa nos planos inclinados foram consideradas apenas as frações que inferem sobre a transmissividade da atmosfera. Foram realizadas correlações entre $\left\langle\mathrm{K}_{\mathrm{TH}}^{\mathrm{d}}\right\rangle \mathrm{e}\left\langle\mathrm{K}_{\mathrm{T} \beta}^{\mathrm{d}}\right\rangle ;\left\langle\mathrm{K}_{\mathrm{TH}}^{\mathrm{d}}\right\rangle \mathrm{e}\left\langle\mathrm{K}_{\mathrm{B} \beta}^{\mathrm{d}}\right\rangle ;\left\langle\mathrm{K}_{\mathrm{TH}}^{\mathrm{d}}\right\rangle$ $\mathrm{e}\left\langle\mathrm{K}_{\mathrm{D} \beta}^{\mathrm{d}^{\mathrm{s}}}\right\rangle$, obtidas respectivamente pelas seguintes relações radiométricas:

$$
\begin{aligned}
& \left\langle\mathrm{K}_{\mathrm{TH}}^{\mathrm{d}}\right\rangle=\frac{\left\langle\mathrm{H}_{\mathrm{GH}}^{\mathrm{d}}\right\rangle}{\left\langle\mathrm{H}_{0 \mathrm{H}}^{\mathrm{d}}\right\rangle} \\
& \left\langle\mathrm{K}_{\mathrm{T} \beta}^{\mathrm{d}}\right\rangle=\frac{\left\langle\mathrm{H}_{\mathrm{G} \beta}^{\mathrm{d}}\right\rangle}{\left\langle\mathrm{H}_{0 \beta}^{\mathrm{d}}\right\rangle} \\
& \left\langle\mathrm{K}_{\mathrm{B} \beta}^{\mathrm{d}}\right\rangle=\frac{\left\langle\mathrm{H}_{\mathrm{B} \beta}^{\mathrm{d}}\right\rangle}{\left\langle\mathrm{H}_{0 \beta}^{\mathrm{d}}\right\rangle} \\
& \left\langle\mathrm{K}_{\mathrm{d} \beta}^{\mathrm{d}}\right\rangle=\frac{\left\langle\mathrm{H}_{\mathrm{D} \beta}^{\mathrm{d}}\right\rangle}{\left\langle\mathrm{H}_{0 \beta}^{\mathrm{d}}\right\rangle}
\end{aligned}
$$

Os modelos de estimativas conceituais que correlacionam as frações da radiação direta baseados no índice de claridade, normalmente são apresentados por meio de ajustes na forma sigmoidal (Oliveira, 2001; Oliveira \& Escobedo, 2001), polinomial de quarto grau (Gomes, 2006; Gomes et al., 2006) ou lineares, quando os valores de $\left\langle\mathrm{K}_{\mathrm{TH}}^{\mathrm{d}}\right\rangle$ são particionados em função da cobertura de céu (Scolar et al., 2003). Neste trabalho foram propostas correlações das frações $\left\langle\mathrm{K}_{\mathrm{B} \beta}^{\prime d}\right\rangle$ e $\left\langle\mathrm{K}_{\mathrm{TH}}^{\mathrm{d}}\right\rangle$, pois as radiações no topo da atmosfera para superfícies horizontais e inclinadas podem ser estimadas ao longo do ano, não necessitando de medidas de outras componentes nos planos horizontais e/ou inclinados. Assim, para os agrupamentos anuais e mensais nas três inclinações estudadas, a radiação direta foi estimada pelo seguinte modelo.

$$
\mathrm{K}_{\mathrm{B} \beta}^{\mathrm{d}}=\sum_{\mathrm{j}=\mathrm{o}}^{\mathrm{N}} \mathrm{a}_{\mathrm{j}}\left|\left\langle\mathrm{K}_{\mathrm{TH}}^{\mathrm{d}}\right\rangle \mathrm{i}\right|^{\mathrm{j}}
$$

em que:

$$
\begin{aligned}
& a_{j} \text { - coeficientes da equação de estimativa; } \\
& N \text { - grau do polinômio ajustado. }
\end{aligned}
$$

$\mathrm{Na}$ avaliação do desempenho das equações de estimativas diárias nas superfícies inclinadas quanto nas horizontais, foram empregados os indicativos estatísticos MBE (Mean Bias Error), RMSE (Root Mean Square Error), e "d” de Willmott (1981), dados por:

$$
M B E=\frac{\sum_{i=1}^{N}(P i-O i)}{N}
$$

$\operatorname{RMSE}=\left[\frac{\sum_{\mathrm{i}=1}^{\mathrm{N}}(\mathrm{Pi}-\mathrm{Oi})^{2}}{\mathrm{~N}}\right]^{\frac{1}{2}}$

$$
d=1-\frac{\sum_{i=1}^{N}(P i-O i)^{2}}{\sum_{i=1}^{N}\left(\left|P^{\prime} i\right|+\left|O^{\prime} i\right|\right)^{2}}
$$

$$
\begin{aligned}
& \text { em que: } \\
& \mathrm{P}_{\mathrm{i}} \text { - valores estimados; } \\
& \mathrm{O}^{\mathrm{i}} \text { - valores medidos; } \\
& \mathrm{N} \text { - número de observações; } \\
& \left|\mathrm{P}^{\prime} \mathrm{i}\right| \text { - valor absoluto da diferença Pi - } \overline{\mathrm{Oi}} \\
& \left|\mathrm{O}^{\prime} \mathrm{i}\right| \text { - valor absoluto da diferença } \mathrm{Oi} \text { - } \overline{\mathrm{Oi}}
\end{aligned}
$$

O indicativo MBE representa o desvio das médias e provê informações quanto à performance do modelo a longo prazo, com valores negativos indicando subestimativa e vice-versa. Quanto menor o valor absoluto de MBE melhor é a performance do modelo testado (Stone, 1993), porém uma superestimativa cancela uma subestimativa. O RMSE é a raiz quadrada do erro quadrático médio e informa sobre o valor real do erro produzido pelo modelo. Todavia, alguns erros de grande proporção na soma podem causar acréscimos significativos nos valores de RMSE, além de não diferenciar superestimativa de subestimativa. Quanto menores os valores obtidos para RMSE melhor performance dos modelos. O índice de ajustamento "d", variando de 0 a 1 , representa o quanto os valores estimados se ajustam com os valores medidos (Willmott, 1981).

A utilização dos indicativos estatísticos MBE, RMSE e índice de ajustamento "d", em conjunto, permitem uma alternativa adequada para validação de modelos estatísticos, com análises simultâneas dos desvios da média, identificando 
a ocorrência de sub ou superestimativa, espalhamento e ajustamento do modelo em relação aos valores medidos.

\section{RESULTADOS E DISCUSSÃO}

Ocorreu um aumento nos espalhamentos das correlações entre $\left\langle\mathrm{K}_{\mathrm{TH}}^{\mathrm{d}}\right\rangle$ e $\left\langle\mathrm{K}_{\mathrm{T} \beta}^{\mathrm{d}}\right\rangle$ em agrupamentos anuais, com o incremento do ângulo de inclinação (Figura 1), resultando em redução do coeficiente de determinação. Este comportamento decorreu do maior nível de radiação refletida e também do ganho de radiação direta com o aumento do ângulo de incidência no período de inverno (Iqbal, 1983).

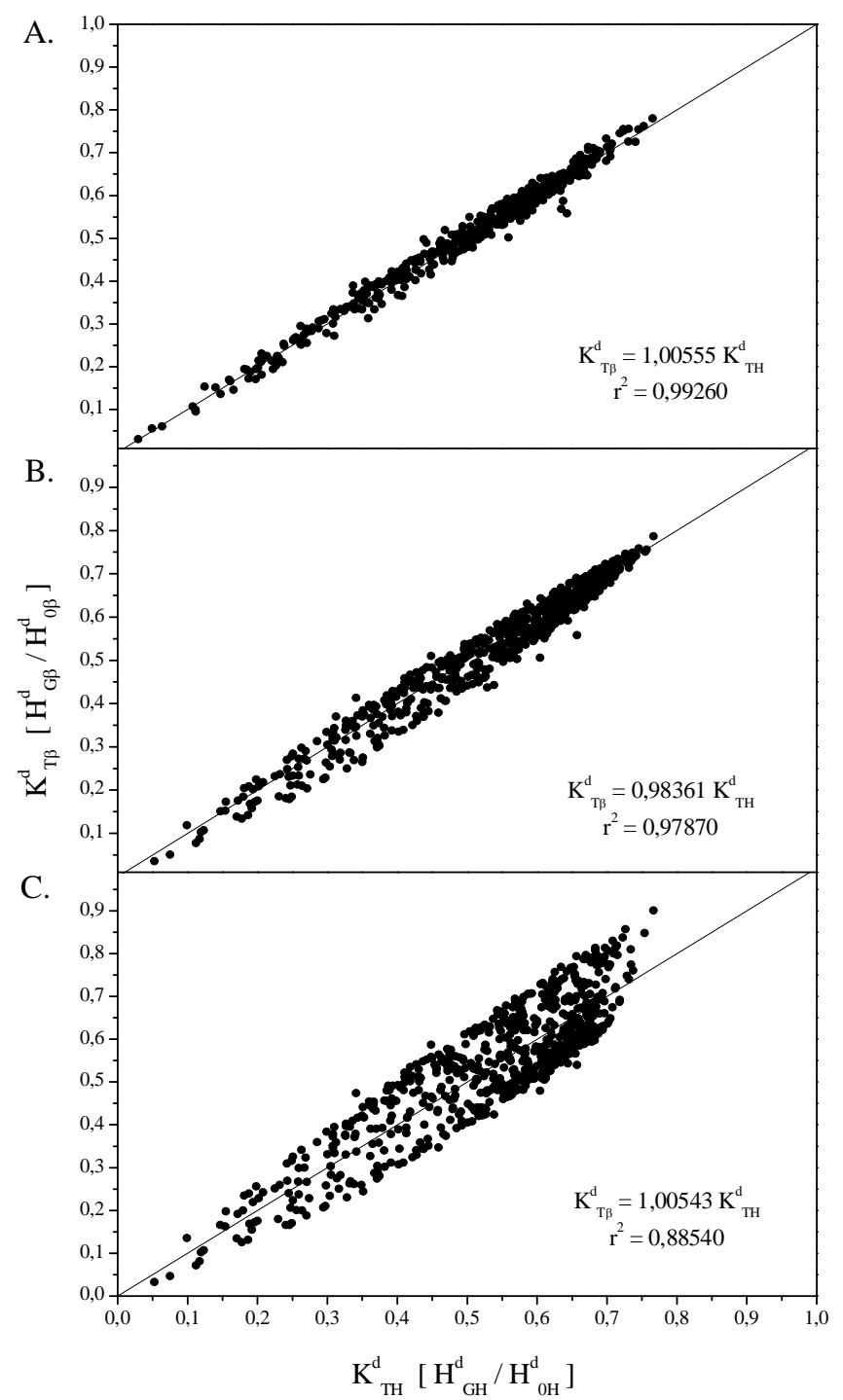

Figura 1. Correlação diária entre o índice de claridade de

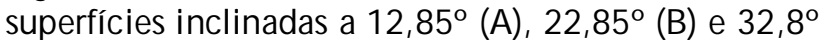
(C) com face para o N orte e o índice de claridade na horizontal, com agrupamento anual de dados

A correlação entre $\left\langle\mathrm{K}_{\mathrm{B} \beta}^{\mathrm{d}}\right\rangle$ e $\left\langle\mathrm{K}_{\mathrm{TH}}^{\mathrm{d}}\right\rangle$ é apresentada na Figura 2; aos agrupamentos anuais com inclinações de $12,85^{\circ}, 22,85^{\circ} \mathrm{e}$ $32,85^{\circ}$ (Figuras $2 \mathrm{~A}, 2 \mathrm{~B}$ e $2 \mathrm{C}$ ), foram ajustadas as seguintes equações de quarto grau, com coeficientes de correlação de 0,$9481 ; 0,9393$ e 0,9663 , respectivamente:

$$
\begin{aligned}
\mathrm{K}_{\mathrm{B} \beta}^{\mathrm{d}}= & -0,20293+2,66318\left\langle\mathrm{~K}_{\mathrm{TH}}^{\mathrm{d}}\right\rangle-11,77585\left\langle\mathrm{~K}_{\mathrm{TH}}^{\mathrm{d}}\right\rangle^{2}+ \\
& +22,07032\left\langle\mathrm{~K}_{\mathrm{TH}}^{\mathrm{d}}\right\rangle^{3}-12,23303\left\langle\mathrm{~K}_{\mathrm{TH}}^{\mathrm{d}}\right\rangle^{4}
\end{aligned}
$$

$$
\begin{aligned}
\mathrm{K}_{\mathrm{B} \beta}^{\mathrm{d}}= & 0,22207-2,82839\left\langle\mathrm{~K}_{\mathrm{TH}}^{\mathrm{d}}\right\rangle+11,48682\left\langle\mathrm{~K}_{\mathrm{TH}}^{\mathrm{d}}\right\rangle^{2}- \\
& -16,36156\left\langle\mathrm{~K}_{\mathrm{TH}}^{\mathrm{d}}\right\rangle^{3}+9,45413\left\langle\mathrm{~K}_{\mathrm{TH}}^{\mathrm{d}}\right\rangle^{4} \\
\mathrm{~K}_{\mathrm{B} \beta}^{\mathrm{d}}= & -0,72687+8,00744\left\langle\mathrm{~K}_{\mathrm{TH}}^{\mathrm{d}}\right\rangle-32,10447\left\langle\mathrm{~K}_{\mathrm{TH}}^{\mathrm{d}}\right\rangle^{2}+ \\
& +56,21064\left\langle\mathrm{~K}_{\mathrm{TH}}^{\mathrm{d}}\right\rangle^{3}-32,55354\left\langle\mathrm{~K}_{\mathrm{TH}}^{\mathrm{d}}\right\rangle^{4}
\end{aligned}
$$

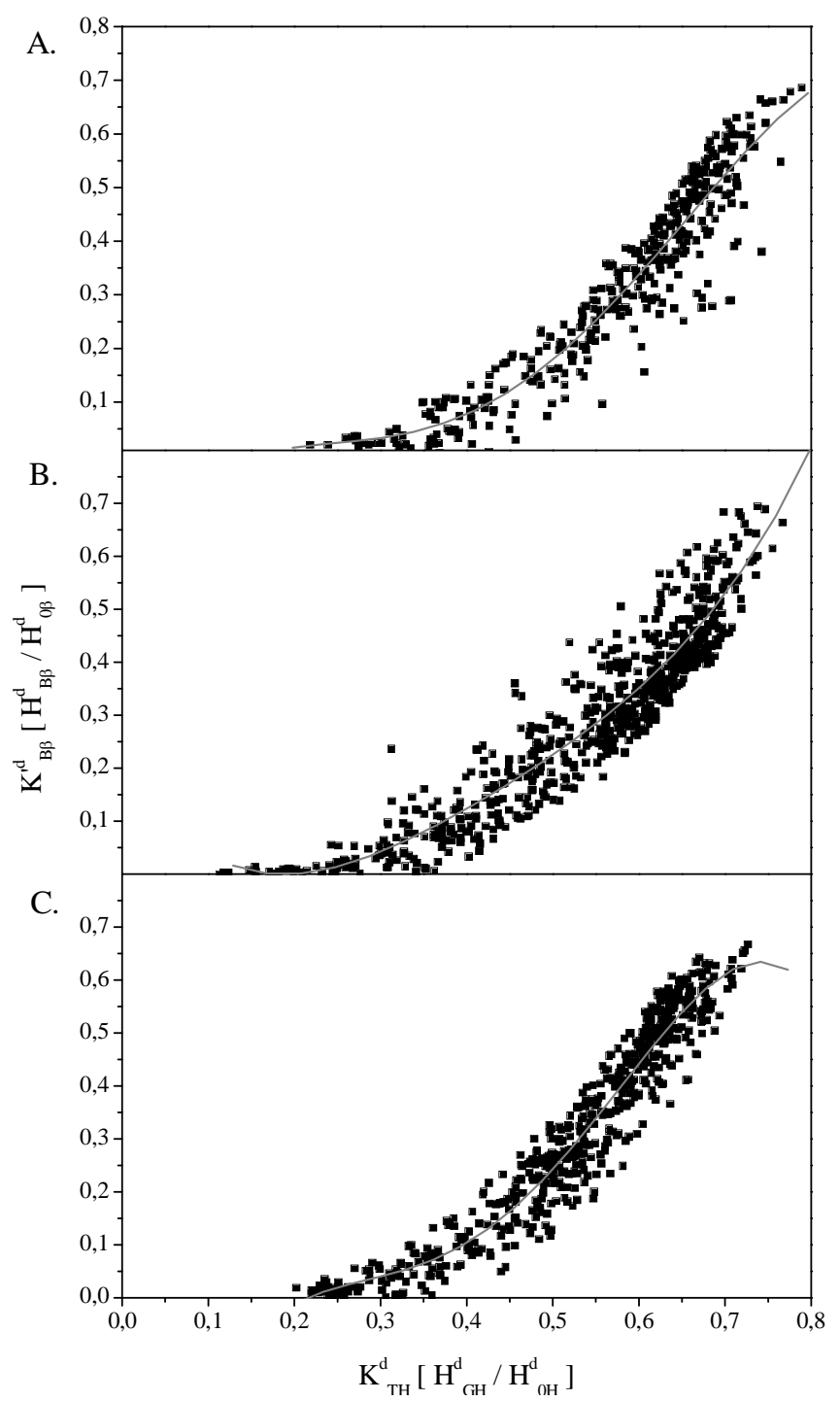

Figura 2. Correlação entre $\left\langle\mathrm{K}_{\mathrm{B} \beta}^{\mathrm{d}}\right\rangle$ para superfícies inclinadas a $12,85^{\circ}$ (A), 22,85응 (B) e 32,85 (C) com a face para o Norte e $\left\langle\mathrm{K}_{\mathrm{TH}}^{\mathrm{d}}\right\rangle$ de superfícies horizontais

Não foi verificada linearidade entre $\left\langle\mathrm{K}_{\mathrm{B} \beta}^{\mathrm{d}}\right\rangle$ e $\left\langle\mathrm{K}_{\mathrm{TH}}^{\mathrm{d}}\right\rangle$, pois para um mesmo valor de $\left\langle\mathrm{K}_{\mathrm{TH}}^{\mathrm{d}}\right\rangle$, podem ser observados diversos valores de $\left\langle\mathrm{K}_{\mathrm{B} \beta}^{\prime \mathrm{d}}\right\rangle$, resultantes do grande espalhamento da correlação. Notou-se, porém, uma concentração de valores de $\left\langle\mathrm{K}_{\mathrm{TH}}^{\mathrm{d}}\right\rangle$ variáveis entre 0,50 e 0,70, resultante do maior número de dias de céu aberto na região (Teramoto et al., 2009; Escobedo 
et al., 2009). Quando os valores de $\left\langle\mathrm{K}_{\mathrm{TH}}^{\mathrm{d}}\right\rangle$ foram inferiores a 0,35 , indicando condições de céu nublado, os níveis de radiação direta nas superfícies inclinadas foram inferiores a $2,7 \%$ do total incidente no topo da atmosfera.

Para o intervalo entre $0,35 \leq\left\langle\mathrm{K}_{\mathrm{TH}}^{\mathrm{d}}\right\rangle \leq 0,65$, os valores de $\left\langle\mathrm{K}_{\mathrm{B} \beta}^{\mathrm{d}}\right\rangle$ aumentaram de 0,05 a 0,$49 ; 0,04$ a 0,$58 ; 0,07$ a 0,61, com médias de 0,$235 ; 0,261$ e 0,315 , para as inclinações de $12,85^{\circ}$, $22,85^{\circ}$ e $32,85^{\circ}$, respectivamente; já nas condições de céu aberto a transmissividade direta atingiu valores médios de 50,7; 49,3 e $56,6 \%$ e valores máximos de 68,$7 ; 69,4$ e $66,7 \%$, para $0,70<$ $\left\langle\mathrm{K}_{\mathrm{TH}}^{\mathrm{d}}\right\rangle \leq 0,78$, nas mesmas inclinações supracitadas. Para valores de $\left\langle\mathrm{K}_{\mathrm{TH}}^{\mathrm{d}}\right\rangle$ acima de 0,78 não se verificaram dependências lógicas, pois os níveis de $\left\langle\mathrm{K}_{\mathrm{B} \beta}^{\mathrm{d}}\right\rangle$ diminuíram com o aumento dos valores de $\left\langle\mathrm{K}_{\mathrm{TH}}^{\mathrm{d}}\right\rangle$. Por conseguinte, tais valores foram eliminados da correlação. Este comportamento decorreu das multirreflexões causadas por nuvens adjacentes, sobretudo em condições de céu parcialmente nublado, quando a fração direta da radiação no topo da atmosfera tende a diminuir. Esses resultados corroboram com os encontrados por Solanki \& Sangani (2008) e El-Sebaii et al. (2010), para superfícies inclinadas para o Sul nas condições climáticas de Jeddah, na Arábia Saudita (latitude $21^{\circ} 42$ ' 37" N).

$\mathrm{Na}$ estimativa da radiação difusa para planos inclinados, também foram adotadas as correlações do tipo Liu \& Jordan (1960) entre as frações $\left\langle\mathrm{K}_{\mathrm{D} \beta}^{\mathrm{d}}\right\rangle \mathrm{e}\left\langle\mathrm{K}_{\mathrm{TH}}^{\mathrm{d}}\right\rangle$, pois dispensam medidas de radiação global inclinada (Figura 3). Inicialmente, a correlação entre $\left\langle\mathrm{K}_{\mathrm{D} \beta}^{\mathrm{d}}\right\rangle$ e $\left\langle\mathrm{K}_{\mathrm{TH}}^{\mathrm{d}}\right\rangle$ apresentou grande espalhamento, dificultando a obtenção de equações de estimativa. A aplicação de curvas médias com valores discretos de $\left\langle\mathrm{K}_{\mathrm{TH}}^{\mathrm{d}}\right\rangle$ para intervalos centesimais (de 0,01 em 0,01 unidades), possibilitou a avaliação da tendência média de variação de $\left\langle\mathrm{K}_{\mathrm{D} \beta}^{\mathrm{d}}\right\rangle$. Em cada um desses subintervalos foram calculados a média de $\left\langle\mathrm{K}_{\mathrm{D} \beta}^{\prime \mathrm{d}}\right\rangle$ e o desvio $\left(\delta \overline{\mathrm{K}}_{\mathrm{D} \beta}^{\prime}\right)$ para as três inclinações (Escobedo et al., 2009).

Para a fração difusa da radiação solar inclinada o comportamento foi descrito por seguintes polinômios de $2^{\circ}$ grau (Eqs. 12 a 14) para os agrupamentos anuais, com coeficientes de determinação iguais a 0,84025; 0,89071 e 0,87502 (Figuras 3A-3C):

$$
\begin{aligned}
& \left\langle\mathrm{K}_{\mathrm{D} \beta}^{\mathrm{d}}\right\rangle=-0,02254+1,22689\left\langle\mathrm{~K}_{\mathrm{TH}}^{\mathrm{d}}\right\rangle-1,49656\left\langle\mathrm{~K}_{\mathrm{TH}}^{\mathrm{d}}\right\rangle^{2} \\
& \left\langle\mathrm{~K}_{\mathrm{D} \beta}^{\mathrm{d}}\right\rangle=-0,02922+1,43189\left\langle\mathrm{~K}_{\mathrm{TH}}^{\mathrm{d}}\right\rangle-1,70392\left\langle\mathrm{~K}_{\mathrm{TH}}^{\mathrm{d}}\right\rangle^{2} \\
& \left\langle\mathrm{~K}_{\mathrm{D} \beta}^{\mathrm{d}}\right\rangle=-0,04254+1,51652\left\langle\mathrm{~K}_{\mathrm{TH}}^{\mathrm{d}}\right\rangle-1,96006\left\langle\mathrm{~K}_{\mathrm{TH}}^{\mathrm{d}}\right\rangle^{2}
\end{aligned}
$$

Essas curvas médias apresentaram crescimento dos valores de $\left\langle\mathrm{K}_{\mathrm{D} \beta}^{\mathrm{d}}\right\rangle$ com pontos máximos de 0,2666; 0,3115 e 0,2917, que correspondem a condições de nebulosidade moderada, cujos valores de $\left\langle\mathrm{K}_{\mathrm{TH}}^{\mathrm{d}}\right\rangle$ variaram entre 0,35 e 0,45 , com decréscimos em torno de 0,$07 ; 0,10$ e 0,05 para condições de pouca nebulosidade, quando $\left\langle\mathrm{K}_{\mathrm{TH}}^{\mathrm{d}}\right\rangle$ se aproxima de 0,75 .

Para dias de céu claro, as maiores contribuições nos níveis de radiação global foram observadas para a componente direta, resultando em níveis inferiores de radiação difusa incidente permitindo, também, um decréscimo dos valores de $\left\langle\mathrm{K}_{\mathrm{D \beta}}^{\mathrm{d}}\right\rangle$. Outros autores verificaram esta tendência quadrática da fração

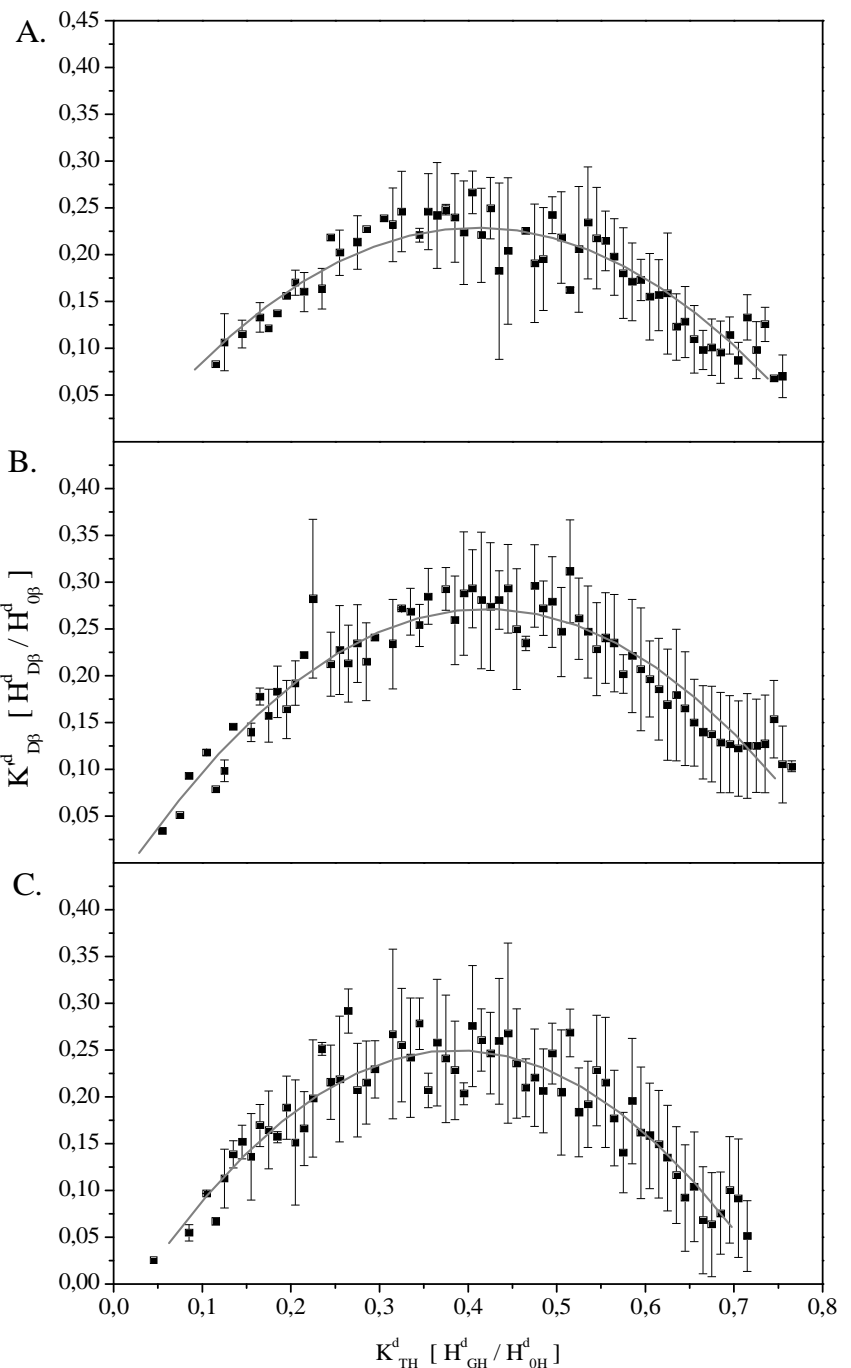

Figura 3. Correlação entre $\left\langle\mathrm{K}_{\mathrm{D} \beta}^{\mathrm{d}}\right\rangle$ e $\left\langle\mathrm{K}_{\mathrm{TH}}^{\mathrm{d}}\right\rangle$ pela curva média com agrupamento anual, para as inclinações de $12,85^{\circ}$ (A), $22,85^{\circ}$ (B) e $32,8^{\circ}$ (C) com a face para 0 Norte

$\left\langle\mathrm{K}_{\mathrm{D} \beta}^{\mathrm{dd}}\right\rangle$ correlacionada com o índice de claridade de superfície horizontal (Jiang, 2009; Posadillo \& Luque, 2009).

A menor variação do desvio-padrão para valores de $\left\langle\mathrm{K}_{\mathrm{TH}}^{\mathrm{d}}\right\rangle$ inferiores a 0,20 (condição de céu nublado) resulta do efeito minimizado da massa ótica para essa condição de cobertura; quando $\left\langle\mathrm{H}_{\mathrm{TH}}^{\mathrm{d}}\right\rangle>0,20$, essa variabilidade não depende exclusivamente dos valores do índice de claridade mas também da massa ótica, que permite gerar o efeito de sazonalidade nos modelos de estimativa com agrupamentos mensais (Scolar et al., 2003; Assunção et al., 2007).

Scolar et al. (2003), apresentaram uma correlação entre a fração difusa da radiação global inclinada e $\left\langle\mathrm{K}_{\mathrm{TH}}^{\mathrm{d}}\right\rangle$, com ajuste de um polinômio de quarto grau, com $\mathrm{R}^{2}=0,91$.

$$
\begin{aligned}
\mathrm{K}_{\mathrm{D} \beta}= & 0,993+0,178\left\langle\mathrm{~K}_{\mathrm{TH}}\right\rangle-0,945\left\langle\mathrm{~K}_{\mathrm{TH}}\right\rangle^{2}- \\
& -4,71\left\langle\mathrm{~K}_{\mathrm{TH}}\right\rangle^{3}+4,89\left\langle\mathrm{~K}_{\mathrm{TH}}\right\rangle^{4}
\end{aligned}
$$

O comportamento quadrático encontrado decorreu do uso da fração $\left\langle\mathrm{K}_{\mathrm{D} \beta}^{\prime d}\right\rangle$ que, no período do inverno (dias com 
nebulosidade alta, decorrentes da entrada de frentes frias), decresce com o aumento da inclinação em função do aumento da radiação no topo da atmosfera no plano inclinado e dos baixos valores do índice de claridade, indicando, assim, níveis baixos de radiação global incidente com grande contribuição da componente difusa.

No desempenho das estimativas da radiação global em superfícies inclinadas com agrupamentos anuais, verificaramse superestimativas dos valores de $\left\langle\mathrm{K}_{\mathrm{T} \beta}^{\mathrm{d}}\right\rangle$, com MBE de 1,93; 5,29 e 7,86\% respectivamente e índices de ajustamento superiores a $87 \%$; os maiores espalhamentos ocorreram com o aumento da inclinação (Tabela 1).

Tabela 1. Validações das correlações entre as frações global, direta e difusa com $\left\langle\mathrm{K}_{\mathrm{TH}}^{\mathrm{d}}\right\rangle$ nos agrupamentos anuais, para as inclinações de 12,85, 22,85 e 32,85응

\begin{tabular}{|c|c|c|c|c|c|c|}
\hline \multirow{2}{*}{ Inclinação } & \multirow{2}{*}{ Correlação } & \multicolumn{2}{|c|}{ MBE } & \multicolumn{2}{|c|}{ RMSE } & \multirow{2}{*}{$\begin{array}{c}\text { Indicativo } \\
\text { "d" }\end{array}$} \\
\hline & & $\%$ & Energia & $\%$ & Energia & \\
\hline 12,850 & $\left\langle\mathrm{~K}_{\mathrm{T \beta}}^{\mathrm{d}}\right\rangle^{\mathrm{X}}\left\langle\mathrm{K}_{\mathrm{TH}}^{\mathrm{d}}\right\rangle$ & 1,93 & 0,394 & 5,81 & 1,186 & 0,9901 \\
\hline $22,85^{\circ}$ & $\left\langle\mathrm{K}_{\mathrm{T} \beta}^{\mathrm{d}}\right\rangle\left\langle\left\langle\mathrm{K}_{\mathrm{TH}}^{\mathrm{d}}\right\rangle\right.$ & 5,29 & 1,025 & 11,39 & 2,205 & 0,9523 \\
\hline $32,85^{\circ}$ & $\left\langle\mathrm{K}_{\mathrm{T}}^{\mathrm{d}}\right\rangle\left\langle\left\langle\mathrm{K}_{\mathrm{TH}}^{\mathrm{d}}\right\rangle\right.$ & 7,86 & 1,485 & 19,76 & 3,733 & 0,8792 \\
\hline $12,85^{0}$ & $\left\langle\mathrm{~K}_{\mathrm{B \beta}}^{\mathrm{d}}\right\rangle^{\mathrm{X}}\left\langle\mathrm{K}_{\mathrm{TH}}^{\mathrm{d}}\right\rangle$ & $-2,12$ & $-0,225$ & 19,93 & 1,798 & 0,9836 \\
\hline $22,85^{0}$ & $\left\langle\mathrm{~K}_{\mathrm{B} \beta}^{\mathrm{d}}\right\rangle^{\mathrm{X}}\left\langle\mathrm{K}_{\mathrm{TH}}^{\mathrm{d}}\right\rangle$ & $-3,57$ & $-0,443$ & 14,56 & 1,805 & 0,9774 \\
\hline 32,850 & $\left.\left\langle\mathrm{~K}_{\mathrm{B} \beta}^{\mathrm{d}}\right\rangle{ }^{\mathrm{d}} \mathrm{X}_{\mathrm{TH}}\right\rangle$ & 3,88 & 0,519 & 13,09 & 1,748 & 0,9854 \\
\hline 12,850 & $\left\langle\mathrm{~K}_{\mathrm{D \beta}}^{\mathrm{d}}\right\rangle^{\mathrm{X}}\left\langle\mathrm{K}_{\mathrm{TH}}^{\mathrm{d}}\right\rangle$ & $-14,68$ & $-1,417$ & 31,83 & 3,188 & 0,6214 \\
\hline 22,850 & $\left\langle\mathrm{~K}_{\mathrm{D} \beta}^{\mathrm{d}}\right\rangle \mathrm{X}\left\langle\mathrm{K}_{\mathrm{TH}}^{\mathrm{d}}\right\rangle$ & 3,09 & 0,194 & 43,43 & 2,731 & 0,6155 \\
\hline 32,850 & $\left\langle\mathrm{~K}_{\mathrm{DB}}^{\mathrm{dd}}\right\rangle^{\mathrm{X}}\left\langle\mathrm{K}_{\mathrm{TH}}^{\mathrm{d}}\right\rangle$ & $-7,51$ & $-0,459$ & 43,65 & 3,885 & 0,6115 \\
\hline
\end{tabular}

As equações de estimativa da radiação direta com agrupamentos anuais da Tabela 1 possibilitaram bons resultados, com tendências a subestimar em até $-3,6 \%(-0,44$ $\left.\mathrm{MJ} \mathrm{m}^{-2} \mathrm{~d}^{-1}\right)$ na inclinação de $22,85^{\circ}$ e superestimar em $0,52 \mathrm{MJ}$ $\mathrm{m}^{-2} \mathrm{~d}^{-1}(32,85 \%)$. Os espalhamentos foram de 19,93; 14,56 e $13,09 \%$, ou 1,$80 ; 1,81$ e $1,75 \mathrm{MJ} \mathrm{m}^{-2} \mathrm{~d}^{-1}$, com ajustamentos de 0,9836; 0,9774 e 0,9854, para as mesmas inclinações, respectivamente. Os modelos estatísticos de estimativa da radiação difusa apresentaram desempenhos ruins, com ajustamentos em torno de $62 \%$ e espalhamentos acima de $30 \%$; todavia, corroboraram com o verificado na literatura para estimativas na partição diária, inclusive quando são utilizados modelos paramétricos na obtenção da radiação global para superfícies inclinadas (Miguel et al., 2001; Kamali et al., 2006; Kudish \& Evseev, 2008; El-Sebaii et al., 2010).

As equações de estimativa com agrupamentos mensais apresentaram comportamentos similares aos agrupamentos anuais, porém para agrupamentos menores (meses), pode-se evidenciar o efeito sazonal ao longo do ano.

Na estimativa da radiação global para superfícies inclinadas, através de equações mensais (Tabela 2), podem ser observados dois períodos distintos que acompanham a evolução da radiação global e no topo da atmosfera, com coeficientes angulares maiores que 1,0 no verão e menores entre os equinócios. Verificaram-se as piores correlações nos meses de abril e agosto, decorrentes da entrada das frentes frias na região, que propicia uma grande variação no número de dias com céu nublado e céu aberto (Teramoto et al., 2009; Escobedo et al., 2009).

Tabela 2. Coeficiente angular e de determinação $\left(R^{2}\right) d a$ regressão linear $\mathrm{K}_{\mathrm{T} \beta}^{\mathrm{d}}=\mathrm{a}_{1}\left\langle\mathrm{~K}_{\mathrm{TH}}^{\mathrm{d}}\right\rangle$ para inclinações de $12,85,22,85$ e $32,85^{\circ}$, com a face para o N orte

\begin{tabular}{|c|c|c|c|c|c|c|}
\hline \multirow{2}{*}{ Mês } & \multicolumn{2}{|c|}{12,850} & \multicolumn{2}{|c|}{ 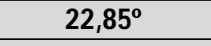 } & \multicolumn{2}{|c|}{32,85} \\
\hline & $a_{1}$ & $\mathbf{R}^{2}$ & $a_{1}$ & $\mathbf{R}^{2}$ & $a_{1}$ & $\mathbf{R}^{2}$ \\
\hline J aneiro & 1,0363 & 0,9966 & 1,0463 & 0,9961 & 1,0947 & 0,9944 \\
\hline Fevereiro & 1,0068 & 0,9969 & 1,0252 & 0,9979 & 1,0485 & 0,9964 \\
\hline Março & 0,9763 & 0,9935 & 0,9929 & 0,9924 & 0,9965 & 0,9946 \\
\hline Abril & 0,9795 & 0,9283 & 0,9743 & 0,9987 & 0,9463 & 0,9880 \\
\hline Maio & 0,9820 & 0,9981 & 0,9443 & 0,9914 & 0,9235 & 0,9949 \\
\hline J unho & 0,9866 & 0,9888 & 0,9311 & 0,9936 & 0,9008 & 0,9924 \\
\hline J ulho & 0,9871 & 0,9968 & 0,9365 & 0,9902 & 0,9089 & 0,9942 \\
\hline Agosto & 0,9909 & 0,9986 & 0,8927 & 0,9006 & 0,9560 & 0,9941 \\
\hline Setembro & 0,9975 & 0,9985 & 0,9721 & 0,9946 & 0,9828 & 0,9957 \\
\hline Outubro & 1,0081 & 0,9944 & 1,0075 & 0,9984 & 1,0239 & 0,9927 \\
\hline Novembro & 1,0289 & 0,9978 & 1,0298 & 0,9981 & 1,0624 & 0,9957 \\
\hline Dezembro & 1,0361 & 0,9978 & 1,0477 & 0,9980 & 1,0928 & 0,9953 \\
\hline
\end{tabular}

Nas validações foram observados efeitos sazonais dos modelos mensais de estimativa da radiação global para superfícies inclinadas, haja vista que as superestimativas ocorreram no período em que o Sol declina no Hemisfério Norte (21/03 a 21/09), enquanto na primavera e no verão os modelos podem ser caracterizados como de superestimativas. As equações anuais da Figura 1, quando aplicadas nos meses apresentaram desempenhos melhores do que as equações mensais (Tabela 2), apenas nos meses de março e setembro (equinócios), que representam os valores médios do agrupamento anual da radiação global nas inclinações. Os piores desempenhos foram verificados nos meses de abril e maio, em função da grande variabilidade da cobertura de céu, decorrente da entrada de frentes frias na região. Nos meses de verão (dezembro e janeiro), as equações citadas na Tabela 2 para as inclinações de 22,85 e $32,85^{\circ}$, subestimaram a radiação global incidente na superfície inclinada, dadas pelos valores de MBE negativos (Figura 4). Kamali et al. (2006) em Karaj (Iran latitude $35^{\circ} 55^{\prime} \mathrm{N}$ ) e El-Sebaii et al. (2010) também observaram comportamentos similares com sazonalidades alternadas em função do hemisfério.

$\mathrm{Na}$ inclinação de $12,85^{\circ}$ foram validados apenas os modelos gerados para os meses de primavera e verão, em função do período de medidas (18 meses) e da base usada na geração das equações (12 meses). Em geral, na inclinação de 32,85\% foram verificados os maiores desvios das médias nas estimativas da $\left\langle\mathrm{H}_{\mathrm{G} \beta}^{\mathrm{d}}\right\rangle$ com equações mensais de inverno e desvios variando entre 1,30 e 1,90 $\mathrm{MJ} \mathrm{m}^{-2} \mathrm{~d}^{-1}$ entre os meses de maio e agosto.

Não se verificou tendência alguma quanto aos sinais dos coeficientes dos polinômios mensais de estimativa da radiação direta nas superfícies inclinadas (Tabela 3); entretanto, para valores de $\left\langle\mathrm{K}_{\mathrm{TH}}^{\mathrm{d}}\right\rangle\left\langle 0,35\right.$, se tem valores menores ainda de $\left\langle\mathrm{K}_{\mathrm{B} \beta}^{\mathrm{d}}\right\rangle$. Os modelos gerados a partir de agrupamentos mensais apresentaram tendência de subestimativas e superestimativas variáveis entre as inclinações.

Para o ângulo de $12,85^{\circ}$ ocorreram subestimativas de radiação direta em janeiro e fevereiro e superestimativas entre setembro e novembro (Figura 5). Desvios acima de $1,0 \mathrm{MJ} \mathrm{m}^{-2} \mathrm{~d}^{-1} \mathrm{em}$ valor absoluto foram encontrados somente no mês de fevereiro; contudo, os maiores espalhamentos foram verificados para o mês de janeiro $\left(2,97 \mathrm{MJ} \mathrm{m}^{-2} \mathrm{~d}^{-1}\right)$. Nesta inclinação todos os modelos apresentaram 


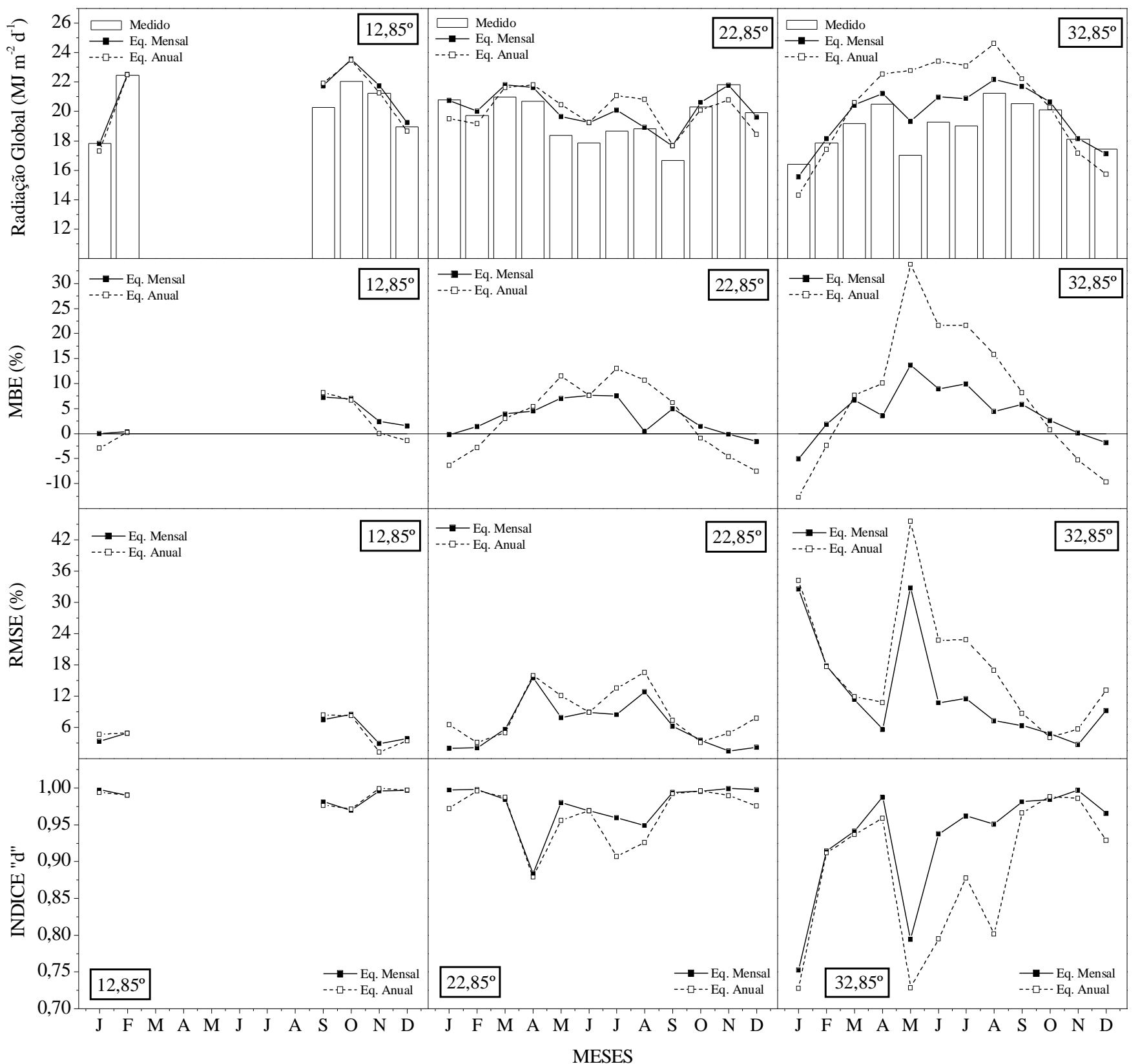

Figura 4. Comportamento das relações entre $\left\langle\mathrm{K}_{\mathrm{T} \beta}^{\mathrm{d}}\right\rangle$ e $\left\langle\mathrm{K}_{\mathrm{TH}}^{\mathrm{d}}\right\rangle$ com agrupamentos mensais e equações mensais e anuais, nas inclinações de 12,85, 22,85 e 32,85은

ajustamentos entre valores medidos e estimados de radiação direta sobre uma superfície inclinada, superiores a $94 \%$.

$\mathrm{Na}$ inclinação de $22,85^{\circ}$, as subestimativas da radiação direta ocorreram em março, abril, maio, agosto e setembro, com MBE variando entre $-1,15 \mathrm{e}-2,81 \mathrm{MJ} \mathrm{m}^{-2} \mathrm{~d}^{-1}$, respectivamente (Figura 5). O mês de abril apresentou o pior desempenho, com espalhamentos da ordem de $23 \%\left(3,41 \mathrm{MJ} \mathrm{m}^{-2} \mathrm{~d}^{-1}\right)$ e ajustamento de 0,93 . No ângulo de $32,85^{\circ}$ ocorreram, em 6 meses, superestimativas de $\left\langle\mathrm{H}_{\mathrm{B} \beta}^{\mathrm{d}}\right\rangle$ com valores máximos de $8,83 \%(1,23$ $\left.\mathrm{MJ} \mathrm{m}^{-2} \mathrm{~d}^{-1}\right)$. Os valores de MBE foram inferiores a $-0,83 \mathrm{MJ} \mathrm{m}^{-2} \mathrm{~d}^{-1}$ (agosto) e em termos percentuais a $-5,55 \%$ (em janeiro). Os maiores espalhamentos dessa radiação foram verificados em fevereiro e março, $2,96 \mathrm{MJ} \mathrm{m}^{-2} \mathrm{~d}^{-1}(28,48 \%)$ e $2,43 \mathrm{MJ} \mathrm{m}^{-2} \mathrm{~d}^{-1}$ $(20,29 \%)$, respectivamente, com ajustamentos superiores a $95 \%$.

Os resultados para a radiação direta em superfícies inclinadas se apresentaram próximos aos obtidos por vários autores que realizaram correlações de estimativa da radiação direta para superfícies horizontais, com base na correlação com o índice de claridade, verificando-se valores de MBE variando entre -1,5 e 13,2\%; RMSE entre 6,7 e 31\% (Battles et al., 2000; Lopez et al., 2000; Gupta \& Ralegaonkar, 2004; Gomes et al., 2006; Islam et al., 2010).

Para a grande maioria dos meses as equações mensais foram melhores para a estimativa da radiação direta, com índice de ajustamento para as equações mensais acima de $97 \%$, indicando que podem ser aplicadas tanto as equações anuais quanto as mensais nesses meses.

O modelo de estimativa com agrupamentos mensais que correlaciona $\left\langle\mathrm{K}_{\mathrm{D} \beta}^{\mathrm{d}}\right\rangle \operatorname{com}\left\langle\mathrm{K}_{\mathrm{TH}}^{\mathrm{d}}\right\rangle$ por equações quadráticas é apresentado na Tabela 4. As piores correlações foram verificadas nos meses de fevereiro, março e julho, com $\mathrm{R}^{2} \mathrm{em}$ torno de 0,80 . 
Tabela 3. Coeficiente de regressão dos model os de estimativa da fração $\left\langle\mathrm{K}_{\mathrm{B} \beta}^{\mathrm{d}}\right\rangle$ em função da fração $\left\langle\mathrm{K}_{\mathrm{TH}}^{\mathrm{d}}\right\rangle$, com agrupamentos mensais e coeficientes de determinação, para as inclinações de 12,85, 22,85 e 32,85, com a face para o N orte

\begin{tabular}{|c|c|c|c|c|c|c|c|}
\hline \multirow[t]{2}{*}{ Inclinação } & \multirow[t]{2}{*}{ Mês } & \multicolumn{5}{|c|}{$\mathrm{K}_{\mathrm{B} \beta}^{\mathrm{d}}=\sum_{\mathrm{i}=0}^{4}\left(\left(\mathrm{~K}_{\mathrm{TH}}^{\mathrm{d}}\right)_{\mathrm{i}}\right)^{\mathrm{j}}$} & \multirow[t]{2}{*}{$\mathbf{R}^{2}$} \\
\hline & & $a_{0}$ & $a_{1}$ & $a_{2}$ & $a_{3}$ & $a_{4}$ & \\
\hline \multirow{12}{*}{$12,85^{0}$} & J aneiro & 1,9399 & $-17,8900$ & 58,1573 & $-77,6346$ & 38,4126 & 0,9428 \\
\hline & Fevereiro & 0,0141 & $-0,2660$ & 1,4304 & $-0,4686$ & 0,6928 & 0,9625 \\
\hline & Março & 1,4085 & $-10,3858$ & 27,8481 & $-32,0076$ & 13,6140 & 0,9307 \\
\hline & Abril & 1,2195 & $-7,6474$ & 17,9503 & $-18,6898$ & 7,2851 & 0,9400 \\
\hline & Maio & $-0,0887$ & 1,3873 & $-6,5893$ & 13,9448 & $-7,6584$ & 0,9564 \\
\hline & J unho & 1,3158 & $-8,5078$ & 20,5188 & $-21,8595$ & 8,6975 & 0,9764 \\
\hline & J ulho & 0,0006 & $-4,7246$ & 11,5992 & $-8,4538$ & 2,1584 & 0,9790 \\
\hline & Agosto & $-0,5104$ & 7,6822 & $-37,1234$ & 71,3693 & $-44,2778$ & 0,9584 \\
\hline & Setembro & $-0,0113$ & 0,2653 & $-1,6435$ & 5,2932 & $-2,7193$ & 0,9618 \\
\hline & Outubro & $-0,2024$ & 1,8526 & $-6,4587$ & 11,4789 & $-5,5941$ & 0,9195 \\
\hline & Novembro & 1,3523 & $-13,7166$ & 48,4054 & $-69,7583$ & 37,4069 & 0,9452 \\
\hline & Dezembro & 0,3056 & $-3,0743$ & 10,2211 & $-11,6668$ & 5,6917 & 0,9558 \\
\hline \multirow{12}{*}{22,850} & J aneiro & $-0,3374$ & 3,7767 & $-15,1769$ & 29,1215 & $-17,7169$ & 0,9401 \\
\hline & Fevereiro & $-0,3680$ & 4,1341 & $-16,8460$ & 31,8475 & $-19,2879$ & 0,9396 \\
\hline & Março & 0,4646 & $-4,5525$ & 14,1645 & $-15,1179$ & 6,2331 & 0,9340 \\
\hline & Abril & $-0,0198$ & 0,6297 & $-4,9885$ & 13,8050 & $-9,3203$ & 0,9256 \\
\hline & Maio & $-0,9373$ & 10,7740 & $-40,1736$ & 65,2439 & $-36,5553$ & 0,9498 \\
\hline & J unho & $-0,3863$ & 5,1785 & $-20,1082$ & 34,5938 & $-19,6957$ & 0,9797 \\
\hline & J ulho & $-0,1145$ & 2,4910 & $-10,3522$ & 19,1467 & $-10,6828$ & 0,9632 \\
\hline & Agosto & $-0,2512$ & 3,5629 & $-15,4237$ & 28,7655 & $-16,8943$ & 0,9783 \\
\hline & Setembro & $-0,1466$ & 1,7614 & $-7,6484$ & 15,4729 & $-8,8976$ & 0,9583 \\
\hline & Outubro & 0,3149 & $-3,4045$ & 11,6509 & $-13,7244$ & 6,7206 & 0,9785 \\
\hline & Novembro & 0,3582 & $-3,6638$ & 11,6015 & $-11,7171$ & 4,7868 & 0,9674 \\
\hline & Dezembro & 0,2853 & $-2,4429$ & 5,9822 & $-1,3347$ & $-1,8457$ & 0,9635 \\
\hline \multirow{12}{*}{32,850} & J aneiro & 0,5059 & $-5,1620$ & 17,4463 & $-21,7767$ & 10,8786 & 0,9513 \\
\hline & Fevereiro & 1,1987 & $-9,8723$ & 27,2453 & $-30,0159$ & 14,2965 & 0,8968 \\
\hline & Março & 1,5762 & $-16,3868$ & 58,6380 & $-85,1301$ & 45,8438 & 0,8880 \\
\hline & Abril & $-0,1388$ & 0,1340 & 0,2077 & 0,7771 & 1,3330 & 0,9351 \\
\hline & Maio & 0,6614 & $-7,6477$ & 26,2191 & $-34,6042$ & 18,0185 & 0,9615 \\
\hline & J unho & $-0,6368$ & 6,4197 & $-27,8155$ & 52,4021 & $-31,8315$ & 0,9334 \\
\hline & J ulho & $-0,4416$ & 4,1555 & $-18,4796$ & 36,0853 & $-21,7610$ & 0,9808 \\
\hline & Agos to & $-0,8749$ & 8,7466 & $-34,8085$ & 60,0273 & $-34,2813$ & 0,9779 \\
\hline & Setembro & $-0,3897$ & 4,5248 & $-18,6009$ & 33,2728 & $-18,4670$ & 0,9635 \\
\hline & Outubro & 0,0253 & 0,1616 & $-2,8525$ & 9,6233 & $-5,9515$ & 0,9426 \\
\hline & Novembro & $-0,6017$ & 7,3631 & $-31,7038$ & 55,4553 & $-31,7505$ & 0,9382 \\
\hline & Dezembro & 0,1571 & $-1,0413$ & 2,7521 & 0,0877 & $-0,9496$ & 0,9055 \\
\hline
\end{tabular}

Tabela 4. Coeficiente de regressão dos modelos de estimativa da fração $\left\langle\mathrm{K}_{\mathrm{D} \beta}^{\mathrm{d}}\right\rangle$ em função da fração $\left\langle\mathrm{K}_{\mathrm{TH}}^{\mathrm{d}}\right\rangle$, com agrupamentos mensais e coeficientes de determinação, para as inclinações de 12,85, 22,85e e 32,85, com a face para o Norte

\begin{tabular}{|c|c|c|c|c|c|c|c|c|c|c|c|c|}
\hline \multirow[t]{2}{*}{ Mês } & \multicolumn{4}{|c|}{$\mathrm{K}_{\mathrm{D} \beta}^{\mathrm{d}}=\sum_{\mathrm{j}=\mathrm{o}}^{2}\left(\left(\mathrm{~K}_{\mathrm{TH}}^{\mathrm{d}}\right)_{\mathrm{i}}\right)^{\mathrm{j}}$} & \multicolumn{4}{|c|}{$\mathrm{K}_{\mathrm{D} \beta}^{\mathrm{d}}=\sum^{2}\left(\left(\mathrm{~K}_{\mathrm{TH}}^{\mathrm{d}}\right)_{\mathrm{i}}\right)^{\mathrm{j}}$} & \multicolumn{4}{|c|}{$\mathrm{K}_{\mathrm{D} \beta}^{\mathrm{d}}=\sum_{\mathrm{i}=0}^{2}\left(\left(\mathrm{~K}_{\mathrm{TH}}^{\mathrm{d}}\right)_{\mathrm{i}}\right)^{\mathrm{j}}$} \\
\hline & $a_{0}$ & $a_{1}$ & $a_{2}$ & $\mathbf{R}^{2}$ & $a_{0}$ & $a_{1}$ & $a_{2}$ & $\mathbf{R}^{2}$ & $a_{0}$ & $a_{1}$ & $a_{2}$ & $\mathbf{R}^{2}$ \\
\hline & \multicolumn{4}{|c|}{ Inclinação - 12,850 } & \multicolumn{4}{|c|}{ Inclinação - 22,85ํำ } & \multicolumn{4}{|c|}{ Inclinação - 32,850 } \\
\hline J an & 0,15448 & 0,59413 & $-0,69286$ & 0,81346 & $-0,04340$ & 1,70539 & & 0,85418 & $-0,01960$ & 1,72014 & $-2,02769$ & 08603 \\
\hline $\mathrm{Fev}$ & & & $-0,75976$ & & & & & & & 1,58118 & $-1,88978$ & \\
\hline Mar & & & $-1,04845$ & & & & & & & & $-1,86191$ & \\
\hline $\mathrm{Abr}$ & & & $-2,54539$ & & & & & & & 4036 & $-1,75558$ & \\
\hline Mai & & & $-1,93393$ & & & & & & & & $-1,50909$ & \\
\hline J un & & & $-7,36165$ & & & & & & & & $-2,17766$ & \\
\hline Jul & -0 & 1,41214 & $-1,76859$ & & & 1,48448 & 609 & & & 379 & $-1,70410$ & \\
\hline Ago & $-0,05$ & 1,34381 & $-1,72665$ & & & 193 & & & & 952 & $-2,30859$ & \\
\hline Set & & 1,54482 & $-1,84645$ & & & & & & & 1,70996 & $-2,11730$ & \\
\hline Out & $-0,07585$ & 1,66250 & $-2,02203$ & & & 1,52706 & & & & 1,79022 & $-2,22681$ & \\
\hline Nov & $-0,07107$ & 1,73430 & $-1,91993$ & & & 1,61370 & $-1,88325$ & & $-0,00867$ & 1,52036 & $-1,87366$ & 0,86698 \\
\hline Dez & $-0,13902$ & 1,96781 & $-2,24672$ & 0,88464 & 0,06565 & 1,13187 & $-1,02416$ & 0,94555 & $-0,04495$ & 1,86822 & $-2,29548$ & 0,89940 \\
\hline
\end{tabular}




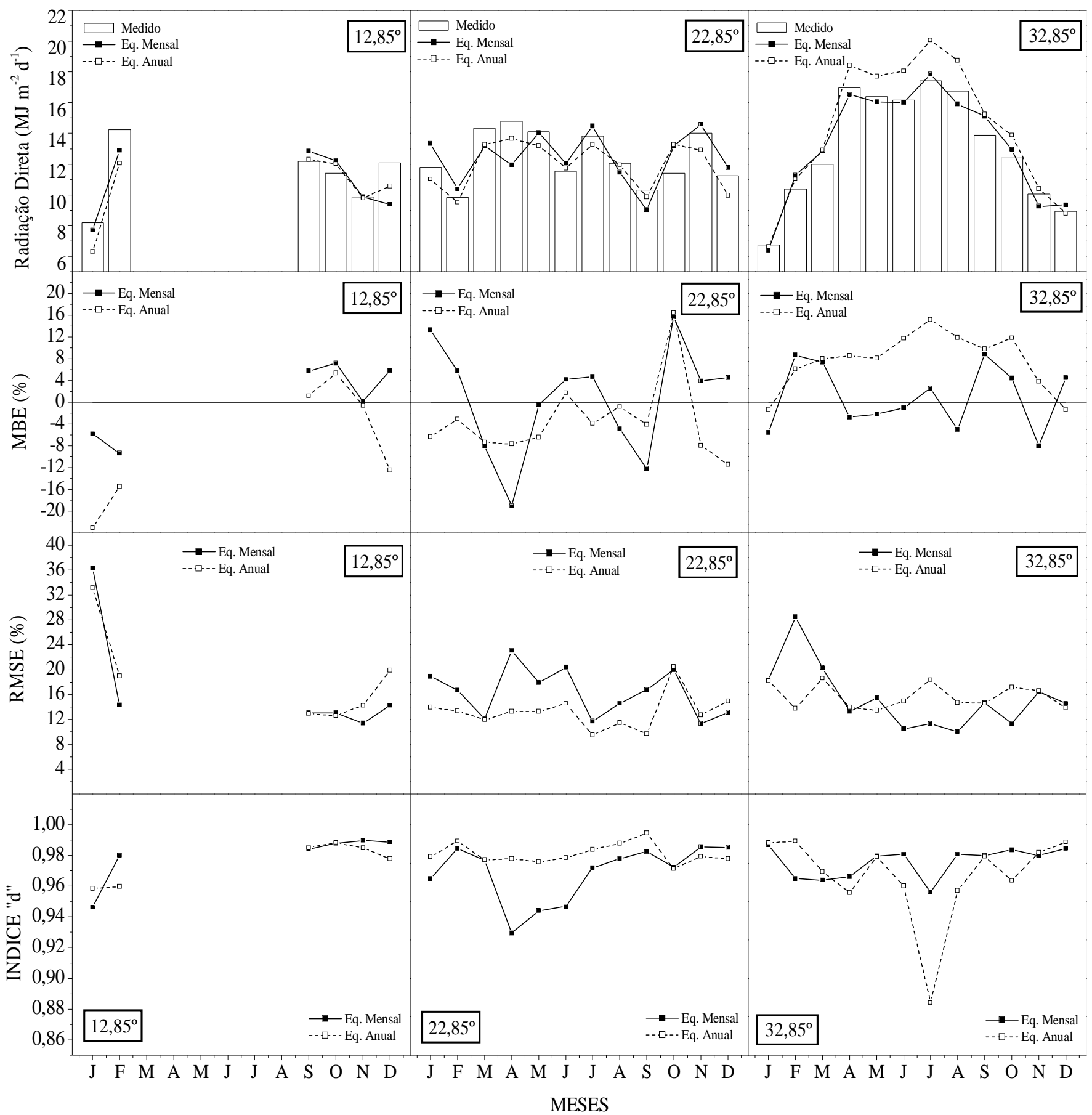

Figura 5. Comportamento das relações entre $\left\langle\mathrm{K}_{\mathrm{B} \beta}^{\mathrm{d}}\right\rangle \mathrm{e}\left\langle\mathrm{K}_{\mathrm{TH}}^{\mathrm{d}}\right\rangle$ com agrupamentos mensais e equações mensais e anuais, para as inclinações de $12,85,22,85$ e 32,85은

Para a grande maioria dos meses da inclinação de $32,85^{\circ}$, o modelo apresentou tendência de subestimar $\left\langle\mathrm{H}_{\mathrm{D} \beta}^{\mathrm{d}}\right\rangle$, com desvios variando entre 0,0006 e $0,9487 \mathrm{MJ} \mathrm{m}^{-2} \mathrm{~d}^{-1}$ ou em entre 0,02 e $12,80 \%$ (Figura 6); já para a inclinação de $22,85^{\circ}$, na maioria dos meses os modelos superestimam os níveis de irradiação difusa incidente entre $0,0521 \mathrm{e}$ $0,9593 \mathrm{MJ} \mathrm{m}^{-2} \mathrm{~d}^{-1}(1,12$ e $12,58 \%)$. Os espalhamentos variaram entre 0,86 e $2,64 \mathrm{MJ} \mathrm{m}^{-2} \mathrm{~d}^{-1}$, correspondentes a 11,99 e $26,56 \%$. Esses resultados estão em conformidade com os verificados na literatura para correlações entre fração difusa inclinada com índice de claridade horizontal, cujos valores de MBE variaram entre -2 a 7,59\%, com RMSE atingindo até 30,94\% (Noorian et al., 2008; Posadillo \& Luque, 2009; Jiang, 2009).

Na maioria dos meses foram encontrados melhores índices de ajustamento para as equações mensais do que os verificados com as equações anuais (Tabela 2), aplicadas nos meses; todavia, os piores valores de "d" foram obtidos para os meses com grande variação da cobertura do céu, como em dezembro, janeiro, fevereiro, maio, junho, julho, setembro e outubro, sendo nos meses de verão, outono/inverno e primavera, a maior influência gerada pela nebulosidade, entrada de frentes frias 


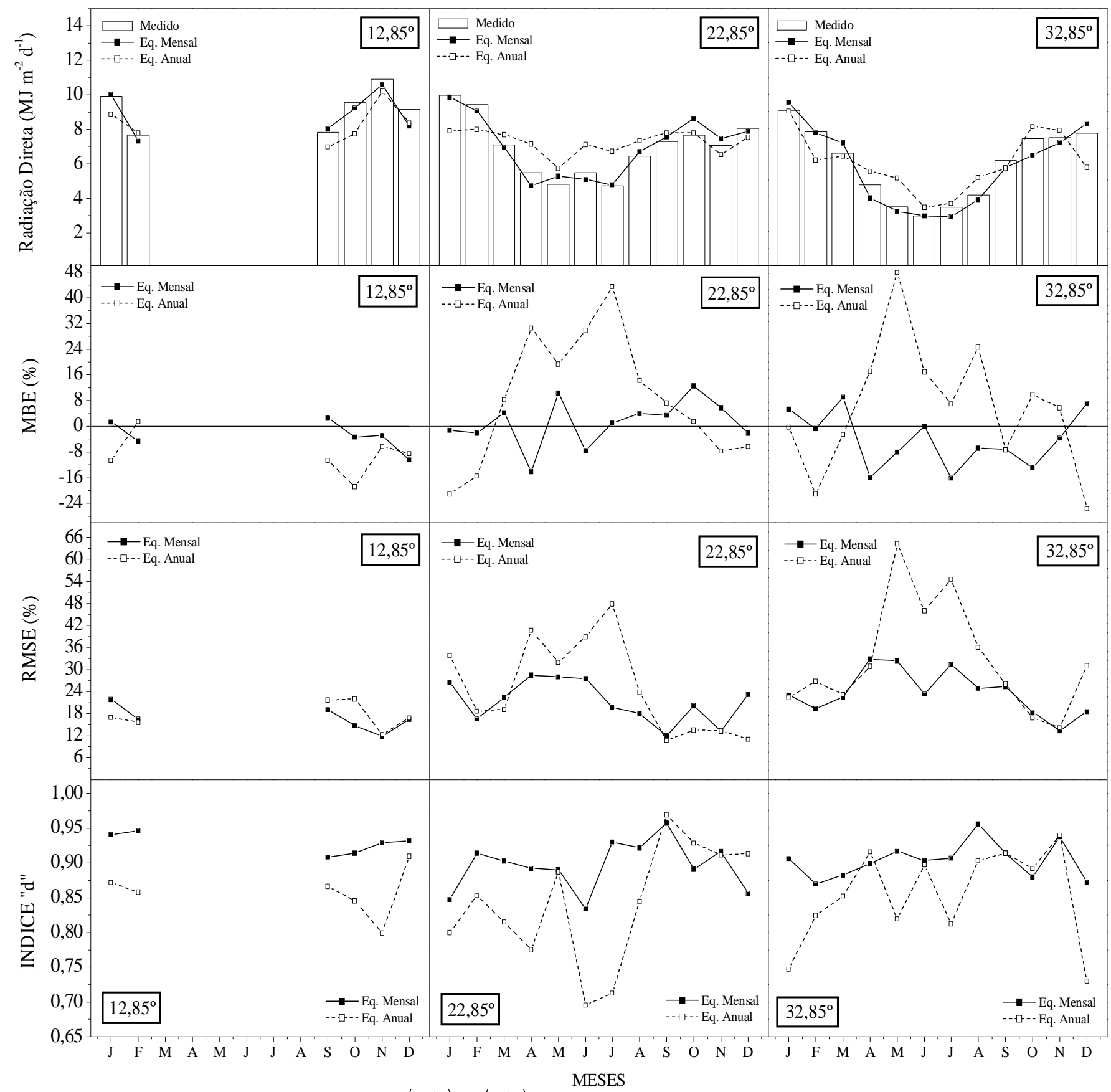

Figura 6. Validações das correlações entre $\left\langle\mathrm{K}_{\mathrm{D} \beta}^{\mathrm{d}}\right\rangle$ e $\left\langle\mathrm{K}_{\mathrm{TH}}^{\mathrm{d}}\right\rangle$ com agrupamentos mensais e equações mensais e anuais, para as inclinações de 12,85으. 22,85 e 32,85응

na região e partículas em suspensão oriundas da queima da cana-de-açúcar na região, respectivamente (Codato et al., 2008; Teramoto et al., 2009).

\section{CONClusÕES}

1. As correlações entre as componentes da radiação solar global, direta e difusa para superfícies inclinadas e a radiação global medida no plano horizontal, apresentaram bons ajustes para estimativas de valores diários, com desempenho decrescente com o aumento do ângulo de inclinação.
2. Para as estimativas das componentes global e direta da radiação solar para superfícies inclinadas pode-se aplicar as equações obtidas nos agrupamentos mensais e anuais, enquanto para a estimativa da radiação difusa as equações mensais possibilitaram melhores resultados.

\section{LITERATURA CITADA}

Assunção, F. H.; Escobedo, J. F.; Oliveira, A. P. A new algorithm to estimate sky condition based on 5 minutes-averaged values of clearness index and relative optical air mass. Theoretical and Applied Climatology, v.90, p.235-248, 2007. 
Battles, F. J.; Rubio, M. A.; Tovar, J.; Olmo, F.J.; AladosArboledas, L. Empirical modeling of hourly direct irradiance by means of hourly global irradiance. Energy, v.25, p.675688, 2000.

Borges, V. P.; Oliveira, A. S.; Coelho Filho, M. A.; Silva, T. S. M.; Pamponet, B. M. Avaliação de modelos de estimativa da radiação solar incidente em Cruz das Almas, Bahia. Revista Brasileira de Engenharia Agrícola e Ambiental, v.14, p.74-80, 2010.

CEPAGRI - Centro de Pesquisas Meteorológicas e Climáticas aplicadas a Agricultura. Clima dos municípios paulistas. http://www.cpa.unicamp.br. 30 Jun. 2009.

Codato, G.; Oliveira, A. P.; Soares, J.; Escobedo, J. F.; Gomes, E. N.; Dal Pai, A. Global and diffuse solar irradiances in urban and rural áreas in Southeast Brazil. Theoretical and Applied Climatology, v.93, p.57-73, 2008.

El-Sebaii, A. A.; Al-Hazmi, F. S.; Al-Ghamdi, A. A.; Yaghmour, S. J. Global, direct and diffuse solar radiation on horizontal and tilted surfaces in Yeddah, Saudi Arabia. Applied Energy, v.87, p.568-576, 2010.

Escobedo, J. F.; Gomes, E. N.; Oliveira, A. P.; Soares, J. Modeling hourly and daily fractions of UV, PAT and NIR to global solar radiation under various Sky conditions at Botucatu, Brazil. Applied Energy, v.86, p.299-309, 2009.

Gomes, E. N. Medidas e modelos de estimativa da radiação direta na incidência. Botucatu: UNESP, 2006. 97p. Tese Doutorado

Gomes, E. N.; Escobedo, J. F.; Oliveira, A. P.; Soares, J. Evolução diurna e anual da radiação direta na incidência. Avances em Energias Renovables y Médio Ambiente, v.10, p.121-129, 2006.

Gueymard, C. A. Direct and indirect uncertainties in the prediction of tilted irradiance for solar engineering applications. Solar Energy, v.83, p.432-444, 2009.

Gupta, R.; Ralegaonkar, R. V. Estimation of beam radiation for optimal orientation and shape decision of buildings in India. Journal of the Institution of Engineers, v.85, p.2732, 2004.

Iqbal, M. An introduction to solar radiation. Canadá: Academic Press, 1983. 390p.

Islam, M. D.; Alili, A. A.; Kubo, I.; Ohadi, M. Measurement of solar-energy (direct beam radiation) in Abu Dhabi, UAE. Renewable Energy, v.35, p.515-519, 2010.

Jiang, Y. Estimation of monthly mean daily diffuse radiation in China. Applied Energy, v.86, p.1458-1464, 2009.

Kamali, G.A.; Moradi, I.; Khalili, A. Estimating solar radiation on tilted surfaces with various orientations: a study case in Karaj (Iran). Theoretical and Applied Climatology, v.84, p.235-241, 2006.

Kudish, A. I.; Evseev, E. G. The assessment of four different correction models applied to the diffuse radiation measured with a shadow ring using global and normal beam radiation measurements for Beer Sheva, Israel. Solar Energy, v.82, p.144-156, 2008.
Liu, B. Y. H.; Jordan, R. C. The interrelationship and characteristic distribution of direct, diffuse and total solar radiation. Solar Energy, v.3, p.01-19, 1960.

Lopez, G.; Rubio, M.A.; Batlles, F.J. Estimation of hourly direct normal from measured global solar irradiance in Spain. Renewable Energy, v.21, n.2, p.171-186, 2000.

Miguel, A. de; Bilbao, J.; Aguiar, R.; Kambezidis, H.; Negro, E. Diffuse solar irradiation model evaluation in the North Mediterranean belt área. Solar Energy, v.70, p.143-153, 2001.

Noorian, A. M.; Moradi, I.; Kamali, G. A. Evaluation of 12 models to estimate hourly diffuse irradiation on inclined surfaces. Renewable Energy, v.33, p.1406-1412, 2008.

Notton, G.; Poggi, P.; Cristofari, C. Predicting hourly solar irradiations on inclined surfaces based on the horizontal measurements: performances of the association of wellknown mathematical models. Energy Conversion and Management, v.47, p.1816-1829, 2006.

Oliveira, L. S. Estimativa da radiação solar direta na incidência nas partições instantânea, horária e diária a partir da radiação solar global. Botucatu: UNESP, 2001. 65p. Dissertação Mestrado

Oliveira, L. S.; Escobedo, J. F. Coeficiente de atenuação da radiação solar direta para Botucatu/SP. Energia na Agricultura, v.16, p.87-92, 2001.

Pereira, A. B.; Villa Nova, N. A.; Escobedo, J. F.; Oliveira, A. $P$. Evaluation of the solar energy potential at surface in São Paulo, SP, Brazil. Revista Brasileira de Agrometeorologia, v.6, p.99-104, 1998.

Posadillo, R.; Luque, R. L. Hourly distributions of the diffuse fraction of global solar irradiation in Córdoba (Spain). Energy Conversion and Management, v.50, p.223-231, 2009.

Scolar, J.; Martins, D.; Escobedo, J. F. Estimativa da irradiação total sobre uma superfície inclinada a partir da irradiação global na horizontal. Revista Brasileira de Geofísica, v.21, p.249-258, 2003.

Silva, R. A.; Silva, V. de P. R. da; Cavalcanti, E. P.; Santos, D. N. Estudo da variabilidade da radiação solar no Nordeste do Brasil. Revista Brasileira de Engenharia Agrícola e Ambiental, v.14, p.501-509, 2010.

Simonson, J. R. The use of weighted $\mathrm{R}_{\mathrm{b}}$ factors in calculating monthly average insolation on tilted surfaces. Solar Energy, v.27, n.5, p.445-447, 1981.

Solanki, C. S.; Sangani, C. S. Estimation of monthly average direct normal solar radiation using elevation angle for any location. Sol Energy Mater Sol Cells, v.92, p.38-44, 2008.

Souza, J. L.; Nicácio, R. M.; Moura, M. A. L. Global solar radiation measurements in Maceió, Brazil. Renewable Energy, v.30, p.1203-1220, 2005.

Stone, R. J. Improved statistical procedure for the evaluation of solar radiation estimation models. Solar Energy, v.51, p.289-291, 1993. 
Teramoto, ET.; Escobedo, J. F.; Gomes, E. N.; Dall'Antonia, L. C.; Souza, A. P. Análise das freqüências de dias de céu aberto e nublado em função das variações climáticas em região de canade-açúcar. In: Congresso Argentino de Meteorologia, 10, e CongressoLatino-Americano e Ibérico de Meteorologia, 13, 2009, Buenos Aires. Anais... Buenos Aires: CAM, 2009. CD-Rom

Tiba, C.; Aguiar, R., Fraidenraich, N. Analysis of a new relationship between monthly global irradiation and sunshine hours from a database of Brazil. Renewable Energy, v.30, p.957-966, 2005.
Turco, J.E.P.; Rizzatti, G.S. Avaliação de modelo matemático para estimar a radiação solar incidente sobre superfícies com diferentes exposições e declividades. Engenharia Agrícola, v.26, p.257-267, 2006.

Wenxian, L.; Wengeng, G.; Shaoxuan, P. U.; Enrong, L. U. Ratios of global radiation on a tilted to horizontal surface for Yunnan Province, China. Energy, v.20, p.723-728, 1995.

Willmott, C. J. On the validation of models. Physical Geography, v.2, p.184-194, 1981. 